\title{
CADASIL: a case report with an atypical presentation and novel mutation
}

\begin{abstract}
Cerebral autosomal dominant arteriopathy with subcortical infarcts and leukoencephalopathy (CADASIL) is a small vessel disease that commonly presents with ischemic episodes, cognitive deficits, migraine with aura, and psychiatric disturbances. It is caused by mutations in the NOTCH3 gene of which several specific point mutations have been identified. We report a 59 year-old female with a confirmed CADASIL diagnosis, an atypical presentation, and an unreported NOTCH3 mutation. She presented with stroke-like symptoms and MRI was initially read as several subacute infarcts throughout the brain. She denied any history of headaches. A review of her imaging suggested CADASIL as a more likely diagnosis. She underwent NOTCH3 testing, which showed a pathogenic p.Arg141Cys mutation and a previously unreported p.Pro68Leu mutation.
\end{abstract}

Volume 4 Issue 2 - 2017

\author{
Brian Spearman, Ajit Karambelkar, Kiran \\ Talekar \\ Department of Radiology, Thomas Jefferson University, USA
}

Correspondence: Brian Spearman, Department of Radiology, Thomas Jefferson University, USA,

Email brian.spearman@jefferson.edu

Received:September II, 2017 | Published: September 20, 2017

Keywords: cadasil, arteriopathy, subcortical, leukoencephalopathy, notch3

Abbreviations: CADASIL, cerebral autosomal dominant arteriopathy with subcortical infarcts and leukoencephalopathy; TIA, transient ischemic attacks; CT, computed tomography; MRI, magnetic resonance imaging; DWI, diffusion weighted imaging; MELAS, mitochondrialencephalopathy and stroke like episodes; EGFr, epidermal growth factor repeat

\section{Introduction}

Cerebral autosomal dominant arteriopathy with subcortical infarcts and leukoencephalopathy (CADASIL) is a heritable small vessel disease that occurs due to a mutation in the NOTCH3 gene. NOTCH3 codes for a large transmembrane receptor that allows for signaling between neighboring cells. The Notch receptor can amplify and consolidate molecular differences, playing a role in cell fate determination. ${ }^{1}$ NOTCH3 has been shown to be a requirement for the generation of functional arteries in mice by regulating arterial differentiation and maturation of vascular smooth muscle cells. ${ }^{2}$

CADASIL is a generalized angiopathy, and the mutated NOTCH3 receptor may be found in the vascular basal lamina of all vessels. However, the vascular complications largely manifest in the white matter of the brain. This discrepancy has been accounted for by several mechanisms. In CADASIL, the NOTCH3 receptor accumulates at the cytoplasmic membrane of vascular smooth muscle cells and pericytes in close vicinity to the disease's characteristic granular osmiophilic deposits. Evidence from mice suggests that this leads to a significant reduction in pericyte number, a progressive loss of astrocytic end-foot process contact with capillaries, disruption of the blood-brain-barrier, and functional impairment of microvessels. ${ }^{2}$

Furthermore, the long penetrating arteries of the brain have been shown to have decreased internal diameters with increased arteriolar wall thickness. These effects are seen most significantly in the white matter, although both gray and white matter is affected. ${ }^{2}$ Initial presentation and disease progression among patients with CADASIL is variable, but age of onset is typically in early or middle adulthood. Migraine with aura is often the first and most commonly reported symptom with a reported mean age of onset of 28 . Strokes or transient ischemic attacks (TIA) may also be the initial symptom with a reported mean age of onset of 41. Less common initial symptoms include depression, cognitive impairment, and seizures. Stroke and TIAs are common and often recurrent, and dementia typically develops. Most patients progress towards severe disability and premature death. ${ }^{3}$

CADASIL is definitively diagnosed by identification of a NOTCH3 mutation on genetic analysis or by the characteristic vascular deposits on skin biopsy. However, the diagnosis may be suspected by clinical signs and symptoms, positive family history for stroke or dementia, or imaging findings on head computed tomography (CT) and magnetic resonance imaging (MRI). Findings on CT are non-specific white matter regions of low attenuation. MRI is the modality of choice and demonstrates widespread confluent white matter hyperintensities. Classically, the initial course of the disease involves the anterior temporal lobe $(86 \%)$ and external capsule $(93 \%)$. Occipital and orbitofrontal subcortical white matter, subcortical U-fibers, and cortex may be relatively spared. Additional findings may include cerebral microhemorrhages (45\%). Subcortical white matter may become diffusely involved later in the course of the disease with eventual cerebral atrophy. ${ }^{3,4}$

\section{Case report}

A 59year-old female presented with altered mental status, rightsided weakness, and dysarthria. She was initially found lying on the couch and was reported to be unresponsive. When she did begin to respond, her speech was nonsensical. Some right-sided weakness was noted, and she was subsequently brought to the hospital for evaluation.

She had a prior hospital admission the previous year for dysarthria and minor right-sided weakness. She was diagnosed at that time with an acute stroke and was discharged on clopidogrel. Her past medical history is otherwise negative, and she does not have stroke risk factors such as hypertension, hyperlipidemia, diabetes, or tobacco use. She recently had a benign head CT after slipping and falling on ice.

Standard MR imaging was performed to evaluate the rightsided weakness in the index patient. It was initially read as faintly 
hyperintense foci on diffusion weighted imaging (DWI), possibly indicating a subacute lacunar infarct in the left basal ganglia, right centrum semiovale, and left anterior and central pons. A stable chronic lacunar infarct was noted in the left genu of the corpus callosum. Labwork revealed the following: cholesterol 154, HDL 88, LDL 45, and triglycerides 106 . Her symptoms resolved during her hospitalization, she was placed on dual anti-platelet therapy, and she was discharged home.

During follow-up in stroke clinic, her imaging was reviewed. MR imaging demonstrated large confluent areas of hyperintense T2 and FLAIR signal abnormality throughout the periventricular and deep white matter with extension into the subcortical white matter (Figure 1) (Figure 2). $\mathrm{T} 1 \mathrm{w}$ images demonstrated low signal, corresponding to the T2/FLAIR abnormality on T2w images. This white matter abnormality is known as leukoaraiosis. The hyperintense T2/FLAIR signal abnormality also involved the anterior temporal lobes (Figure 3) (Figure 4) and external capsules (Figure 5) (Figure 6). This feature on MRI is uncharacteristic of other white matter diseases, notably the sporadic subcortical atherosclerotic encephalopathy. In our patient, the corpus callosum was spared. The lacunar infarcts were small vessel infarcts, typically seen in the deep white matter, basal ganglia, thalami, and the pons. On diffusion images, there was a small area of diffusion restriction in the left corona radiata that was consistent with an acute infarct (Figure 7) (Figure 8). Subsequently, there was an area of encephalomalacia on follow-up MRI at the location of the previous acute infarct. This abnormality correlated with the rightsided weakness seen prior to the index patient's admission.

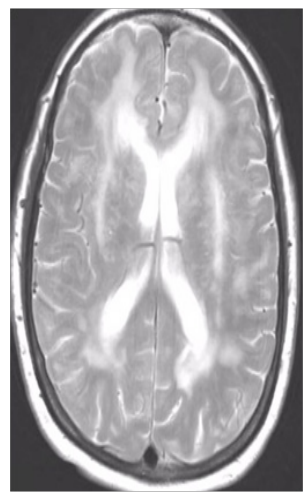

Figure I T2w axial image at the level of corona radiata demonstrates confluent hyperintense signal abnormality in the deep and subcortical cerebral white matter.

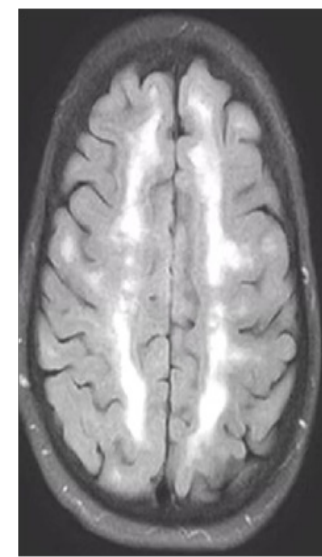

Figure 2 FLAIR axial image at a higher level demonstrates more conspicuous hyperintense white matter signal abnormality.

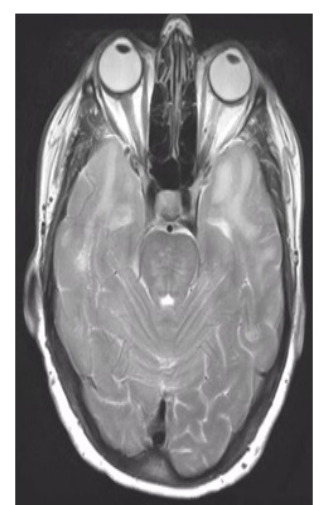

Figure 3 T2 axial image demonstrates hyperintense white matter signal involving the anterior temporal lobes on both sides.

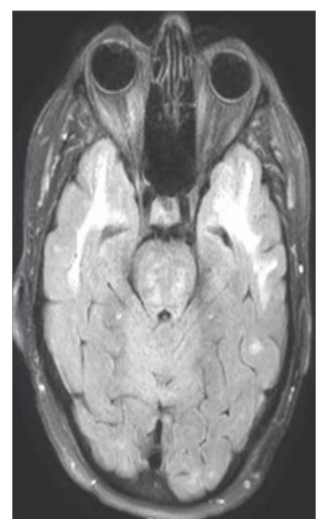

Figure 4 FLAIR axial image demonstrates hyperintense signal in white matter in anterior temporal lobes, on both sides which is more conspicuously seen compared to T2 signal seen on Figure 3.

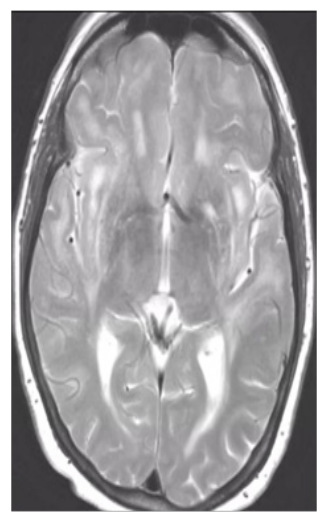

Figure 5 Relatively symmetric hyperintense T2 signal involving the externa capsules, on both sides.

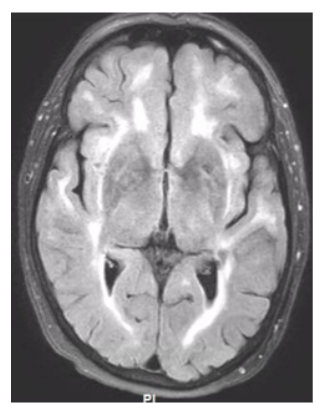

Figure 6 Axial FLAIR image demonstrates relatively symmetric hyperintense signal in external capsules on both sides. 


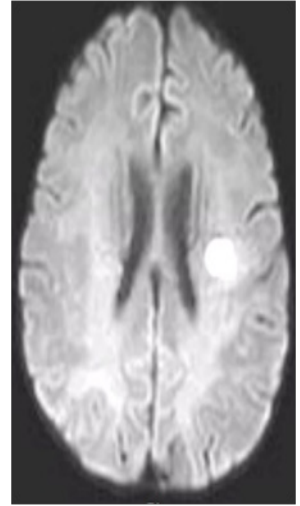

Figure $7 \mathrm{~A}$ small area of diffusion restriction in left frontal corona radiata consistent with an acute infarct.

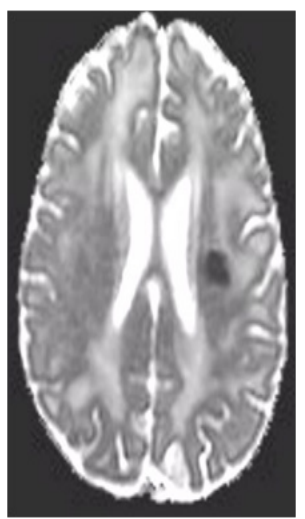

Figure $8 \mathrm{~A}$ small area of diffusion restriction in left corona radiata in left frontal corona radiata consistent with an acute infarct.

The white matter signal abnormality, microbleeds, and lacunar infarcts seen in this patient raised suspicion of CADASIL. Additional history was obtained and included the passing of her father from a stroke at an early age, a lack of headaches, and mild short-term memory problems, including forgetting conversations and repeating herself. She subsequently underwent genetic testing of the NOTCH3 gene, which was positive for heterozygous missense p.Arg141Cys and p.Pro68Leu mutations.

\section{Discussion}

This case of CADASIL is unique for several reasons. The index patient's initial presentation was atypical, the imaging findings were not initially recognized, and the patient was found to have a previously unreported mutation in the NOTCH3 gene. ${ }^{5-7}$

This patient's first presenting symptom of CADASIL occurred at the age of 58 and consisted of an episode of dysarthria and minor right-sided weakness. She was diagnosed with an acute stroke, her symptoms resolved during the admission, and she was discharged on clopidogrel. She had a similar episode one year later. During the interval period, her husband had noticed some short-term memory dysfunction. She denied experiencing migraine with or without aura, and no other cognitive dysfunction had been detected. This late age of onset is nearly two standard deviations above the mean age of onset of 37. Additionally, the mean age of onset for stroke and TIA as a presenting symptom is 41 , again making her nearly 2 standard deviations above the mean. Her lack of migraine is also relatively uncommon with approximately half of all patients reporting migraine throughout the course of the disease. This atypical presentation and rarity of the disease may have contributed to decreased suspicion of CADASIL.

The white matter signal abnormality is nonspecific and seen in many diseases. However, the involvement of the anterior temporal lobes $(86 \%)$ and external capsules $(93 \%)$ is specific to suggest the diagnosis of CADASIL in appropriate clinical settings. ${ }^{8,9}$ CADASIL is a rare entity, its clinical features are nonspecific, and it is almost never considered as a leading differential at first presentation. However, MRI findings are relatively specific in the early course of the disease. MRI helps to rule out large territory infarctions and major intracranial or neck vessel occlusions. It also differentiates other conditions such as mitochondrialencephalopathy and stroke like episodes (MELAS), migraine disorders, and demyelinating disease like multiple sclerosis. Early involvement of the anterior temporal lobes and external capsules is characteristic in the initial stages of CADASIL. As the disease progresses, there is involvement of the white matter, which can also be seen with advanced demyelinating disease and microangiopathy. Hence, radiologists should be aware of the characteristic MR findings and clinical features as it guides the clinician to genetic testing for the specific diagnosis of the CADASIL. ${ }^{10}$

After the determination that imaging findings were consistent with a diagnosis of CADASIL, the patient underwent genetic testing of the NOTCH3 gene. This analysis revealed two mutations: p.Arg141Cys and p.Pro68Leu. Genetic investigation of NOTCH3 mutations have revealed a molecular mechanism to the toxic accumulation of the receptor. NOTCH3 consists of 34 epidermal growth factor repeat (EGFr) domains, each with an even number of cysteine residues. Mutations that disrupt the disulfide bridges between cysteine residues have been proven to cause CADASIL. Genetic analysis of our patient has revealed one such p.Arg141Cys mutation that has been previously identified in CADASIL patients. However, the other p.Pro68Leu mutation does not correlate with this mechanism. It is possible that this mutation may have affected this patient's unique presentation, and further investigation of this mutation may be warranted.

\section{Acknowledgements}

None.

\section{Conflict of interest}

Author declares that there is no conflict of interest.

\section{References}

1. Artavanis-Tsakonas S, Rand MD, Lake RJ. Notch signaling: cell fate control and signal integration in development. Science. 1999;284(5415):770-776.

2. Domenga V, Fardoux P, Lacombe $P$, et al. Notch3 is required for arterial identity and maturation of vascular smooth muscle cells. Genes Dev. 2004;18(22):2730-2735.

3. Joutel A, Andreux F, Gaulis S, et al. The ectodomain of the Notch3 receptor accumulates within the cerebrovasculature of CADASIL patients. J Clin Invest. 2000;105(5):597-605.

4. Ghosh M, Balbi M, Hellal F, et al. Pericytes are involved in the pathogenesis of cerebral autosomal dominant arteriopathy with subcortical infarcts and leukoencephalopathy. Ann Neurol. 2015;78(6):887-900.

5. Miao Q, Paloneva T, Tuominen S, et al. Fibrosis and stenosis of the long penetrating cerebral arteries: the cause of the white matter pathology in cerebral autosomal dominant arteriopathy with subcortical infarcts and leukoencephalopathy. Brain Pathol. 2004;14(4):358-364. 
6. David W Desmond, Joan T Moroney, Timothy Lynch, et al. The natural history of CADASIL. Stroke. 1999;30(6):1230-1233.

7. Opherk C, Peters N, Herzog J, et al. Long-term prognosis and causes of death in CADASIL: a retrospective study in 411 patients. Brain. 2004;127(11):2533-2539.

8. Yousry TA, Seelos K, Mayer M, et al. Characteristic MR lesion pattern and correlation of T1 and T2 lesion volume with neurologic and neuropsychological findings in cerebral autosomal dominant arteriopathy with subcortical infarcts and leukoencephalopathy (CADASIL). AJNR Am J Neuroradiol. 1999;20(1):91-100.
9. Auer DP, Pütz B, Gössl C, et al. Differential lesion patterns in CADASIL and sporadic subcortical arteriosclerotic encephalopathy: MR imaging study with statistical parametric group comparison. Radiology. $2001 ; 218(2): 443-451$

10. Rutten JW, Haan J, Terwindt GM, et al. Interpretation of NOTCH3 mutations in the diagnosis of CADASIL. Expert Rev Mol Diagn. 2014;14(5):593-603. 